\title{
A General Organocatalyst for Direct $\alpha$-Functionalization of Aldehydes: Stereoselective C-C, C-N, C-F, C-Br and C-S Bond-Forming Reactions. Scope and Mechanistic Insights
}

\author{
Johan Franzén, Mauro Marigo, Doris Fielenbach, Tobias C. Wabnitz, Anne Kjarsgaard and Karl \\ Anker Jørgensen*
}

The Danish National Research Foundation: Center for Catalysis, Department of Chemistry, Aarhus University, DK-8000 Aarhus C, Denmark

E-mail: kaj@chem.au.dk

\section{Supporting Information}

\section{Contents}

Experimental Section $\quad$ S2

Computational Methods $\quad$ S5

$\begin{array}{ll}\text { References } & \mathrm{S} 12\end{array}$ 


\section{Experimental Section}

General Methods. The ${ }^{1} \mathrm{H}$ NMR and ${ }^{13} \mathrm{C}$ NMR spectra were recorded at $400 \mathrm{MHz}$ and $100 \mathrm{MHz}$, respectively. The chemical shifts are reported in ppm relative to $\mathrm{CHCl}_{3}(\delta=7.26)$ for ${ }^{1} \mathrm{H} \mathrm{NMR}$ and relative to the central $\mathrm{CDCl}_{3}$ resonance $(\delta=77.0)$ for ${ }^{13} \mathrm{C}$ NMR. Flash chromatography (FC) was carried out using Merck silica gel 60 (230-400 mesh). Optical rotation was measured on a Perkin-Elmer 241 polarimeter.

Materials. All the aldehydes $\mathbf{4 a - i}$, the azodicarboxylates $\mathbf{5}$ and $N$-fluorobenzensulfonimide (NFSI) are commercially available and used as received. Methyl vinyl keton 9, aldehydes $\mathbf{4 a}$ and $\mathbf{4 f}$ were distilled prior too use. The organocatalyst $\mathbf{3},{ }^{1}$ aldehydes $\mathbf{4 j - 1},{ }^{2} \alpha$-imino ethyl glyoxylate $\mathbf{7}^{3}$ and 4,4-dibromo-2,6di-tert-butyl-cyclohexa-2,5-dienone $\mathbf{1 3}^{4}$ were prepared according to published procedure. All solvents were of p.a. quality and used without further purification.

General Procedure for the $\alpha$-Amination of Aldehydes. Azodicarboxylate $5(0.2 \mathrm{mmol})$ was added to a solution of aldehyde $4(0.24 \mathrm{mmol})$ and catalyst $(S)-3 \mathrm{c}(12 \mathrm{mg}, 0.02 \mathrm{mmol})$ in $\mathrm{CH}_{2} \mathrm{Cl}_{2}(0.1 \mathrm{~mL})$ at room temperature. After $20 \mathrm{~min}$, the reaction mixture was diluted with $\mathrm{MeOH}(0.5 \mathrm{~mL})$ followed by careful addition of $\mathrm{NaBH}_{4}(12 \mathrm{mg}, 0.3 \mathrm{mmol})$. After $20 \mathrm{~min}, \mathrm{NaOH}_{\mathrm{aq}}(2.5 \mathrm{~mL} 0.5 \mathrm{M})$ was added and after an additional $2 \mathrm{~h}$ the resulting mixture was diluted with water. The aqueous phase was extracted with $\mathrm{CH}_{2} \mathrm{Cl}_{2}(3 \times 20 \mathrm{~mL})$ and the combined organic phases were dried $\left(\mathrm{Na}_{2} \mathrm{SO}_{4}\right)$. The solvent was removed in vacuo followed by FC purification to give $N$-amino-oxazolidinone 6. Separation conditions of the enantiomers and spectral data for compounds 6a-e were in accordance with those previously reported. ${ }^{5}$ Optical rotation for $\mathbf{6 a - e}$ were of opposite sign to those reported with L-proline. ${ }^{5}$

General Procedure for the Mannich Reaction with Aldehydes. $\alpha$-Imino ethyl glyoxylate 7 (100 $\mathrm{mg}, 0.48 \mathrm{mmol})$ was added to a solution of aldehyde $4(0.96 \mathrm{mmol})$ and catalyst $(S)-3 \mathrm{c}(30 \mathrm{mg}, 0.05$ mmol) in $\mathrm{CH}_{3} \mathrm{CN}(1.25 \mathrm{~mL})$. The resulting mixture was stirred at room temperature for $16 \mathrm{~h}$ and then diluted with water. The aqueous phase was extracted with $\mathrm{CH}_{2} \mathrm{Cl}_{2}(3 \times 20 \mathrm{~mL})$ and the combined organic phases were dried $\left(\mathrm{Na}_{2} \mathrm{SO}_{4}\right)$. The solvent was removed in vacuo followed by FC purification to give the Mannich adduct $\mathbf{8}$.

Compound 8a: ${ }^{1} \mathrm{H}$ NMR $\delta 9.66(d, J=2.5 \mathrm{~Hz}, 1 \mathrm{H}), 6.77(d, J=9.0 \mathrm{~Hz}, 2 \mathrm{H}), 6.65(d, J=9.0 \mathrm{~Hz}$, 2H), $4.27(d d, J=7.1,6.1 \mathrm{~Hz}, 1 \mathrm{H}), 4.17(q, J=7.3 \mathrm{~Hz}, 2 \mathrm{H}) 3.74(s, 3 \mathrm{H}), 2.68(m, 1 \mathrm{H}), 1.76(m, 1 \mathrm{H})$, 
$1.66(m, 1 \mathrm{H}), 1.22(t, J=7.3 \mathrm{~Hz}, 3 \mathrm{H}), 1.01(t, J=7.2 \mathrm{~Hz}, 3 \mathrm{H}) .{ }^{13} \mathrm{C} \mathrm{NMR} \delta 202.8,172.7,153.5,140.7$, $116.3,115.1,61.8,58.3,55.9,55.6,18.8,14.4,12.3$. The enantiomers were separated according to literature procedures. ${ }^{6}[\alpha]^{25}=-43.3\left(\mathrm{c}=1.0, \mathrm{CHCl}_{3}\right)$.

Compound 8b: Separation conditions of the enantiomers, spectral data and optical rotation was in accordance with those previously reported. ${ }^{7}$

Compounds 8c: The title compound was isolated as it corresponding diol after $\mathrm{LiAlH}_{4}$ reduction by the following procedure: The crude reaction mixture was diluted with dry THF $(10 \mathrm{~mL})$ and cooled to 0 ${ }^{\circ} \mathrm{C}$ followed by careful addition of $\mathrm{LiAlH}_{4}(30 \mathrm{mg}, 0.76 \mathrm{mmol})$. After $2 \mathrm{~h}$ at ambient temperature, $\mathrm{NH}_{4} \mathrm{Cl}_{\text {sat }}(20 \mathrm{~mL})$ was carefully added, and the aqueous phase was extracted with $\mathrm{CH}_{2} \mathrm{Cl}_{2}(3 \times 20 \mathrm{~mL})$. The combined organic phases were dried $\left(\mathrm{Na}_{2} \mathrm{SO}_{4}\right)$ and the solvent was removed in vacuo followed by FC purification to give 2-allyl-3-(4-methoxy-phenylamino)-butane-1,4-diol in 63\% yield (two steps). ${ }^{1} \mathrm{H}$ NMR $\delta 6.78(d, J=9.2 \mathrm{~Hz}, 2 \mathrm{H}), 6.69(d, J=9.2 \mathrm{~Hz}, 2 \mathrm{H}), 5.79(m, 1 \mathrm{H}), 5.08(b s, 1 \mathrm{H}), 5.05(b s, 1 \mathrm{H})$, 3.81-3.70 (overlapping peaks including $s$ at $3.75 \mathrm{ppm}$, total $6 \mathrm{H}), 3.66(d d, J=11.0,3.9 \mathrm{~Hz}, 1 \mathrm{H}), 3.48$ $(d d, J=9.5,4.7 \mathrm{~Hz}, 1 \mathrm{H}), 2.23(d t t, J=14.0,6.5,1.3 \mathrm{~Hz}, 1 \mathrm{H}), 2.12(d t t, J=14.0,8.0,1.3 \mathrm{~Hz}, 1 \mathrm{H}), 1.94$ ( $m, 1 \mathrm{H}) .{ }^{13} \mathrm{C}$ NMR $\delta 153.7,140.5,136.4,117.4,117.3,115.2,64.1,62.2,60.5,56.0,42.0,33.9$. The enantiomers were separated by HPLC on Daicel Chiralpak AD with hexane/i-PrOH (95:5) as the eluent: $\mathrm{R}_{\mathrm{t}}$ (min): 44.7 (minor enantiomer); 46.5 (major enantiomer). $[\alpha]_{\mathrm{D}}^{25}=-33.2\left(\mathrm{c}=1.5, \mathrm{CHCl}_{3}\right.$ ).

Compounds 8d: ${ }^{1} \mathrm{H}$ NMR $\delta 9.75(d, J=1.3 \mathrm{~Hz}, 1 \mathrm{H}), 7.25(m, 5 \mathrm{H}), 6.76(d, J=8.9 \mathrm{~Hz}, 2 \mathrm{H}), 6.55(d$, $J=8.9 \mathrm{~Hz}, 2 \mathrm{H}), 4.20(m, 1 \mathrm{H}), 4.22-4.05$ (overlapping peaks, total $4 \mathrm{H}), 3.74(s, 3 \mathrm{H}), 3.30(m, 1 \mathrm{H}), 3.14$ $(d d, J=14.4,7.2 \mathrm{~Hz}, 1 \mathrm{H}), 2.93(d d, J=14.4,7.2 \mathrm{~Hz}, 1 \mathrm{H}), 1.22(t, J=7.2 \mathrm{~Hz}, 3 \mathrm{H}) .{ }^{13} \mathrm{C}$ NMR $\delta 202.7$, $172.5,153.3,140.8,138.1,129.3,129.0,127.1,116.0,115.0,61.9,57.6,55.9,55.5,31.9,25.5 .[\alpha]^{25}=$ -15.2 $\left(\mathrm{c}=1.0, \mathrm{CHCl}_{3}\right)$. The enantiomeric excess was determent after $\mathrm{LiAlH}_{4}$ reduction to the corresponding diol, according to the procedure described for compound 8c, to give 2-benzyl-3-(4methoxy-phenylamino)-butane-1,4-diol in 65\% yield. ${ }^{1} \mathrm{H}$ NMR $\delta$ 7.30-713 (overlapping peaks, total $5 \mathrm{H}), 6.77(d, J=8.8 \mathrm{~Hz}, 2 \mathrm{H}), 6.62(d, J=8.8 \mathrm{~Hz}, 2 \mathrm{H}), 3.79-3.64$ (overlapping peaks including $s$ at 3.73 ppm, total $8 \mathrm{H}), 2.77(d d, J=14.0,8.2 \mathrm{~Hz}, 1 \mathrm{H}), 2.64(d d, J=14.0,8.2 \mathrm{~Hz}, 1 \mathrm{H}), 2.17(m, 1 \mathrm{H}) .{ }^{13} \mathrm{C} \mathrm{NMR}$ $\delta 153.3,140.8,138.11,129.3,129.0,127.1,115.9,115.0,61.9,57.6,55.9,55.5,31.9$, 14.4. The enantiomeric excess was determent by HPLC on Daicel Chiralpak AD with hexane $/ i$-PrOH (95:5) as the eluent: $\mathrm{R}_{\mathrm{t}}$ (min): 48.5 (major enantiomer); 54.0 (minor enantiomer). $[\alpha]^{25}{ }_{\mathrm{D}}=-4.7\left(\mathrm{c}=0.5, \mathrm{CHCl}_{3}\right)$.

Compound 8e: ${ }^{1} \mathrm{H}$ NMR $\delta 9.72(d, J=1.3 \mathrm{~Hz}, 1 \mathrm{H}), 6.77(d, J=9.0 \mathrm{~Hz}, 2 \mathrm{H}), 6.65(d, J=9.0 \mathrm{~Hz}$, 2H), $4.34(d, J=5.9 \mathrm{~Hz}, 1 \mathrm{H}), 4.14(d, J=7.4 \mathrm{~Hz}, 1 \mathrm{H}), 3.73(s, 3 \mathrm{H}), 2.87(t d, J=7.1,5.9 \mathrm{~Hz}, 1 \mathrm{H}), 1.22$ $(t, J=7.4 \mathrm{~Hz}, 3 \mathrm{H}), 1.16(d, J=7.1 \mathrm{~Hz}, 3 \mathrm{H}) .{ }^{13} \mathrm{C} \mathrm{NMR} \delta 201.7,171.7,153.1,140.1,115.6,114.7,61.5$, 
58.6, 55.6, 48.4, 14.1, 9.8. The enantiomers were separated according to literature procedures. ${ }^{6}$

General Procedure for the Michael Addition to Aldehydes. Methyl vinyl ketone 9 (82 $\mu \mathrm{L}, 1.0$ mmol) was added to a solution of aldehyde $4(2.0 \mathrm{mmol})$ and catalyst $(S)-3 \mathbf{c}(60 \mathrm{mg}, 0.1 \mathrm{mmol})$ in EtOH $(0.5 \mathrm{~mL})$ and stirred at $40{ }^{\circ} \mathrm{C}$ for $64 \mathrm{~h}$. The crude reaction mixture was purified by FC, without prior evaporation of the solvent, to give 2-substituted 5-oxo-hexanal 10. Separation conditions of the enantiomers, spectral data and optical rotation for compounds 10a-c were in accordance with those previously reported. ${ }^{8}$

General procedure for the $\alpha$-Bromination of Aldehydes. 4,4-Dibromo-2,6-di-tert-butyl-cyclohexa2,5-dienone 13 (136 mg, $0.38 \mathrm{mmol}$ ) was added to a solution of aldehyde 4 (0.25 mmol), catalyst $(S)$-3c $(0,05 \mathrm{mmol})$ benzoic acid $(6 \mathrm{mg}, 0.05 \mathrm{mmol})$ and water $(10 \mu \mathrm{L})$ in $\mathrm{CH}_{2} \mathrm{Cl}_{2}(3 \mathrm{~mL})$ at $-24{ }^{\circ} \mathrm{C}$. After 90 min at ambient temperature, the reaction mixture was diluted with $\mathrm{MeOH}(1 \mathrm{~mL})$ followed by careful addition of $\mathrm{NaBH}_{4}(15 \mathrm{mg}, 0.38 \mathrm{mmol})$. The mixture was stirred for $10 \mathrm{~min}$, quenched with sat. $\mathrm{NH}_{4} \mathrm{Cl}$ and extracted with $\mathrm{CH}_{2} \mathrm{Cl}_{2}(3 \times 20 \mathrm{~mL})$. The combined organic phases were dried $\left(\mathrm{Na}_{2} \mathrm{SO}_{4}\right)$ and the solvent was removed in vacuo followed by FC purification to give the 2-bromo-alcohol 14. Separation conditions of the enantiomers, spectral data and optical rotation for compounds $\mathbf{1 4 a - b}$ were in accordance with those previously reported. ${ }^{9}$

Compound 14c: ${ }^{1} \mathrm{H}$ NMR $\delta 3.96(d d, J=9.5,2.9 \mathrm{~Hz}, 2 \mathrm{H}), 3.80(d d, J=12.6,9.5 \mathrm{~Hz}, 1 \mathrm{H}), 2.00(m$, $3 \mathrm{H}), 1.68(m, 12 \mathrm{H}) .{ }^{13} \mathrm{C}$ NMR $\delta 76.2,63.2,40.2,36.7,29.7,28.4 .[\alpha]^{25}{ }_{\mathrm{D}}=-0.7\left(\mathrm{c}=0.9 \mathrm{CHCl}_{3}\right)$. The ee was determined after conversion to the corresponding $m$-nitrobenzoyl ester by the following procedure: To a solution of bromo alcohol $14 \mathrm{c}(30 \mathrm{mg}, 0.12 \mathrm{mmol})$ and $\mathrm{Et}_{3} \mathrm{~N}(49 \mu \mathrm{L}, 0.35 \mathrm{mmol})$ was added $\mathrm{m}$ nitrobenzoylchloride (65 $\mathrm{mg}, 0.35 \mathrm{mmol}$ ) and the resulting mixture was stirred at ambient temperature for $20 \mathrm{~min}$. Water was added, and the aqueous phase was extracted with $\mathrm{CH}_{2} \mathrm{Cl}_{2}(3 \times 20 \mathrm{~mL})$ and the combined organic phases were dried $\left(\mathrm{Na}_{2} \mathrm{SO}_{4}\right)$. The solvent was removed in vacuo followed by $\mathrm{FC}$ purification to give 4-nitro-benzoic acid 2-adamantan-1-yl-2-bromo-ethyl ester in 76\% yield. ${ }^{1} \mathrm{H}$ NMR $\delta$ $8.90(s, 1 \mathrm{H}), 8.44(d, J=8.3 \mathrm{~Hz}, 1 \mathrm{H}), 8.40(d, J=7.7 \mathrm{~Hz}, 1 \mathrm{H}), 7.68(t, J=8.1 \mathrm{~Hz}, 1 \mathrm{H}), 4.88(d d, J=$ $11.8,3.1 \mathrm{~Hz}, 1 \mathrm{H}), 4.60(d d, J=11.8,9.0 \mathrm{~Hz}, 1 \mathrm{H}), 4.05(d d, J=9.0,3.1 \mathrm{~Hz}, 1 \mathrm{H}), 2.04(m, 3 \mathrm{H}), 1.72(m$, $12 \mathrm{H}) .{ }^{13} \mathrm{C}$ NMR $\delta 166.4,148.6,135.6,131.9,130.0,127.9,125.0,66.7,66.0,40.2,36.9$, 28.6. The enantiomers were separated by HPLC on Daicel Chiralpak AD with hexane/i-PrOH (90:10) as the eluent: $\mathrm{R}_{\mathrm{t}}$ (min): 12.5 (minor enantiomer); 14.9 (major enantiomer). $[\alpha]^{25}=+54.3$ (c=1.4, $\mathrm{CHCl}_{3}$ ). 


\section{Computational Methods}

All quantum chemical calculations are carried out by using the Gaussian $98^{10}$ or Gaussian $03^{11}$ suite of programs. Using Gaussian98, ${ }^{10}$ initial structures are computed at the HF/6-31G level of theory and are used as starting geometries for calculations which are computed at the B3LYP/6-31G(d) level of theory.

Full geometry optimization and frequency analysis has been performed for all stationary points, and minima structures are characterized by exactly zero imaginary vibrations.

Absolute energies and cartesian coordinates of fully gas phase optimized geometries at the B3LYP/6$31 \mathrm{G}(\mathrm{d})$ level of theory are listed below.

\section{Enamine formed by 3-metyl butanal (4b) and catalyst $(S)-3 \mathrm{c}$}

Charge $=0$

Multiplicity $=1$

Stoichiometry $=\mathrm{C}_{29} \mathrm{H}_{31} \mathrm{NOF}_{12} \mathrm{Si}$

$\mathrm{E}_{\text {elec }}($ gas phase $)=-2741.40305633$ Hartree
$\begin{array}{llll}\mathrm{N} & -1.715905 & 2.262085 & 0.859121\end{array}$
C $\quad-0.299992 \quad 2.116686 \quad 0.521989$
C $\quad 0.399286 \quad 3.148464 \quad 1.441821$
$\begin{array}{llll}\text { C } & -0.541609 & 3.295959 & 2.649296\end{array}$
$\begin{array}{llll}\text { C } & -1.937407 & 3.075865 & 2.055221\end{array}$
$\begin{array}{llll}\text { C } & 0.244452 & 0.638319 & 0.701245\end{array}$
C $\quad 1.607841 \quad 0.432850 \quad-0.000841$
C $\quad 2.084193 \quad-0.873436-0.168025$
C $\quad 3.339994 \quad-1.126254 \quad-0.719296$
$\begin{array}{llll}\text { C } & 4.147381 & -0.075637 & -1.149046\end{array}$
$\begin{array}{llll}\text { C } & 3.671636 & 1.227925 & -1.019434\end{array}$
C $\quad 2.420473 \quad 1.481286 \quad-0.450443$
$\begin{array}{llll}\text { C } & 3.799125 & -2.557507 & -0.821963\end{array}$
F $\quad 3.889289 \quad-3.124970 \quad 0.407046$
$\begin{array}{llll}\text { C } & 4.543747 & 2.385457 & -1.435218\end{array}$
F $\quad 5.309566 \quad 2.818734 \quad-0.408035$
C $\quad-2.686475 \quad 2.254970 \quad-0.141042$
$\begin{array}{llll}\text { C } & -3.978787 & 2.599447 & -0.000582\end{array}$ 

$\begin{array}{llll}\text { C } & -5.015417 & 2.619511 & -1.103870\end{array}$
$\begin{array}{llll}\text { C } & -5.724268 & 3.988696 & -1.140017\end{array}$
$\begin{array}{llll}\text { C } & -0.789657 & -0.341144 & 0.116917\end{array}$
$\begin{array}{llll}\text { C } & -0.894596 & -0.554748 & -1.264171\end{array}$
$\begin{array}{llll}\text { C } & -1.856186 & -1.424051 & -1.778559\end{array}$
$\begin{array}{llll}\text { C } & -2.722755 & -2.111693 & -0.925311\end{array}$
$\begin{array}{llll}\text { C } & -2.624453 & -1.895241 & 0.444840\end{array}$
$\begin{array}{llll}\text { C } & -1.680394 & -1.002734 & 0.959609\end{array}$
$\begin{array}{llll}\text { C } & -3.515842 & -2.623669 & 1.415484\end{array}$
$\begin{array}{llll}\text { F } & -2.780668 & -3.281842 & 2.346451\end{array}$
$\begin{array}{llll}\text { C } & -2.003296 & -1.593222 & -3.267542\end{array}$
$\begin{array}{llll}\text { F } & -2.342158 & -2.858179 & -3.595942\end{array}$
$\begin{array}{llll}\text { O } & 0.367889 & 0.413724 & 2.098609\end{array}$
Si $1.285163 \quad-0.478176 \quad 3.204353$
$\begin{array}{llll}\text { C } & 1.159077 & -2.348289 & 2.950123\end{array}$
$\begin{array}{llll}\text { C } & 3.092689 & 0.064001 & 3.209016\end{array}$
$\begin{array}{llll}\text { C } & 0.487714 & -0.035350 & 4.852517\end{array}$
$\begin{array}{lllll}\text { F } & -4.303753 & -3.532577 & 0.806599\end{array}$
$\begin{array}{llll}\mathrm{F} & -4.320130 & -1.773955 & 2.089925\end{array}$
F $\quad-2.975481-0.787684-3.761602$
$\begin{array}{lllll}\mathrm{F} & -0.865925 & -1.288812 & -3.928324\end{array}$
$\begin{array}{llll}\text { F } & 5.379927 & 2.046528 & -2.438611\end{array}$
F $\quad 3.808748 \quad 3.440165 \quad-1.851304$
F $\quad 5.007642 \quad-2.667348 \quad-1.406432$
F $\quad 2.928212-3.306751 \quad-1.531310$
$\begin{array}{llll}\text { C } & -4.472562 & 2.260829 & -2.493247\end{array}$
$\begin{array}{llll}\mathrm{H} & -4.348478 & 2.889840 & 0.981828\end{array}$
$\begin{array}{llll}\mathrm{H} & -2.312903 & 1.926829 & -1.106198\end{array}$
$\mathrm{H} \quad-2.414263 \quad 4.026353 \quad 1.766143$
$\mathrm{H} \quad-0.452820 \quad 4.275029 \quad 3.129825$
$\begin{array}{llll}\mathrm{H} & 1.405203 & 2.849290 & 1.742630\end{array}$
$\begin{array}{llll}\mathrm{H} & -0.158235 & 2.370004 & -0.536913\end{array}$
$\begin{array}{llll}\mathrm{H} & 0.477141 & 4.100881 & 0.902988\end{array}$ 


$\begin{array}{lrrr}\mathrm{H} & -0.318428 & 2.528548 & 3.392553 \\ \mathrm{H} & -2.615455 & 2.575215 & 2.758180 \\ \mathrm{H} & 2.094261 & 2.509912 & -0.376778 \\ \mathrm{H} & 5.118086 & -0.269029 & -1.588993 \\ \mathrm{H} & 1.463559 & -1.710727 & 0.129947 \\ \mathrm{H} & -0.216233 & -0.056896 & -1.948937 \\ \mathrm{H} & -3.448596 & -2.810623 & -1.323167 \\ \mathrm{H} & -1.630115 & -0.824346 & 2.025868 \\ \mathrm{H} & 3.182413 & 1.153954 & 3.284634 \\ \mathrm{H} & 3.601733 & -0.367177 & 4.080706 \\ \mathrm{H} & 3.640117 & -0.259328 & 2.318032 \\ \mathrm{H} & 0.975121 & -0.576618 & 5.672812 \\ \mathrm{H} & 0.567459 & 1.035901 & 5.069228 \\ \mathrm{H} & -0.575802 & -0.300362 & 4.867873 \\ \mathrm{H} & 1.801556 & -2.722878 & 2.146695 \\ \mathrm{H} & 1.480478 & -2.849472 & 3.872670 \\ \mathrm{H} & 0.130888 & -2.670826 & 2.749662 \\ \mathrm{H} & -5.786548 & 1.871925 & -0.851008 \\ \mathrm{H} & -6.541712 & 3.990991 & -1.871552 \\ \mathrm{H} & -6.148239 & 4.243439 & -0.161291 \\ \mathrm{H} & -5.018183 & 4.782374 & -1.413646 \\ \mathrm{H} & -4.030150 & 1.259614 & -2.521959 \\ \mathrm{H} & -5.279540 & 2.285475 & -3.234343 \\ \mathrm{H} & -3.707258 & 2.979264 & -2.814124\end{array}$

\section{$\underline{\text { Iminium ion intermediate }(S, S)-19}$}

Charge $=1$

Multiplicity $=1$

Stoichiometry $=\mathrm{C}_{29} \mathrm{H}_{31} \mathrm{NOF}_{13} \mathrm{Si}$

$E_{\text {elec }}($ gas phase $)=-2841.03040784$ Hartree

C $\quad 2.360152-1.524250 \quad-0.800422$

C $\quad 1.615061 \quad-0.527006-0.180761$ 

$\begin{array}{llll}\text { C } & 2.304713 & 0.442332 & 0.539863\end{array}$
$\begin{array}{llll}\text { C } & 3.686227 & 0.415132 & 0.634652\end{array}$
$\begin{array}{llll}\text { C } & 4.422262 & -0.572176 & 0.004637\end{array}$
$\begin{array}{llll}\text { C } & 3.742843 & -1.540169 & -0.713562\end{array}$
C $\quad 0.073929-0.537880 \quad-0.159480$
$\begin{array}{llll}\text { O } & -0.385774 & -1.241975 & 0.988567\end{array}$
Si $0.081116 \quad-1.650471 \quad 2.636700$
$\begin{array}{llll}\text { C } & -1.476266 & -2.546210 & 3.255685\end{array}$
$\begin{array}{llll}\text { C } & 4.353582 & 1.457594 & 1.470122\end{array}$
$\begin{array}{llll}\text { F } & 5.704246 & 1.428838 & 1.353174\end{array}$
$\begin{array}{llll}\text { C } & 4.489782 & -2.602895 & -1.450185\end{array}$
$\begin{array}{llll}\text { F } & 3.860871 & -3.812237 & -1.351088\end{array}$
$\begin{array}{llll}\text { C } & -0.516732 & 0.880163 & -0.149502\end{array}$
$\begin{array}{llll}\text { C } & -0.043342 & 1.880329 & -0.993495\end{array}$
$\begin{array}{llll}\text { C } & -0.625800 & 3.136446 & -1.000218\end{array}$
$\begin{array}{llll}\text { C } & -1.679541 & 3.439522 & -0.152553\end{array}$
$\begin{array}{llll}\text { C } & -2.139464 & 2.452432 & 0.698187\end{array}$
$\begin{array}{llll}\text { C } & -1.569128 & 1.188474 & 0.698087\end{array}$
$\begin{array}{llll}\text { C } & -3.244101 & 2.713875 & 1.666218\end{array}$
$\begin{array}{llll}\text { F } & -3.840031 & 3.915008 & 1.468979\end{array}$
$\begin{array}{llll}\text { C } & -0.133851 & 4.137270 & -1.992489\end{array}$
F $\quad-0.560478 \quad 5.394796 \quad-1.719426$
$\begin{array}{llll}\text { C } & -0.497772 & -1.381215 & -1.353382\end{array}$
$\begin{array}{llll}\text { C } & -0.180420 & -0.942583 & -2.791305\end{array}$
$\begin{array}{llll}\text { C } & -1.401282 & -0.161135 & -3.309384\end{array}$
$\begin{array}{llll}\text { C } & -2.591956 & -0.583825 & -2.445961\end{array}$
$\begin{array}{llll}\mathrm{N} & -1.998501 & -1.399395 & -1.325347\end{array}$
$\begin{array}{llll}\text { C } & -2.635587 & -2.089355 & -0.465186\end{array}$
$\begin{array}{llll}\text { C } & -4.114845 & -2.218388 & -0.293187\end{array}$
F $\quad-4.764619-1.193080-1.016090$
$\begin{array}{llll}\text { C } & -4.667705 & -3.583878 & -0.749097\end{array}$
$\begin{array}{llll}\text { C } & -4.006041 & -4.742284 & 0.012945\end{array}$
$\begin{array}{llll}\text { F } & -4.213754 & 1.750963 & 1.580228\end{array}$ 


$$
\begin{array}{llll}
\mathrm{F} & -2.798844 & 2.680758 & 2.957587 \\
\mathrm{~F} & -0.572325 & 3.825542 & -3.253519 \\
\mathrm{~F} & 1.226821 & 4.158793 & -2.059347 \\
\mathrm{~F} & 4.572801 & -2.326738 & -2.785338 \\
\mathrm{~F} & 5.756676 & -2.756968 & -0.990516 \\
\mathrm{~F} & 4.054741 & 1.290317 & 2.795445 \\
\mathrm{~F} & 3.931434 & 2.711537 & 1.141340 \\
\mathrm{C} & 1.574335 & -2.823236 & 2.605803 \\
\mathrm{C} & 0.417914 & -0.086270 & 3.661400 \\
\mathrm{C} & -6.191480 & -3.600611 & -0.566541 \\
\mathrm{H} & -4.343915 & -2.048695 & 0.750561 \\
\mathrm{H} & -2.042679 & -2.642757 & 0.231564 \\
\mathrm{H} & -3.292045 & -1.206215 & -2.975928 \\
\mathrm{H} & -1.586151 & -0.386233 & -4.349583 \\
\mathrm{H} & 0.724244 & -0.358432 & -2.850383 \\
\mathrm{H} & -0.370421 & 0.651080 & 3.564577 \\
\mathrm{H} & -0.182220 & -2.395616 & -1.172864 \\
\mathrm{H} & -0.020863 & -1.826313 & -3.395813 \\
\mathrm{H} & -1.244973 & 0.903756 & -3.241826 \\
\mathrm{H} & -3.127695 & 0.246594 & -2.022084 \\
\mathrm{H} & -1.939402 & 0.443894 & 1.368688 \\
\mathrm{H} & -2.120443 & 4.414931 & -0.153615 \\
\mathrm{H} & 0.790990 & 1.696944 & -1.640605 \\
\mathrm{H} & 1.888981 & -2.311779 & -1.349646 \\
\mathrm{H} & -1.350490156 \\
\mathrm{H} & 1.770263 & 1.228395 & 1.033043 \\
\mathrm{H} & -1.668791 & -3.452929 & 2.691568 \\
\mathrm{H} & -2.836470 & 4.293501 \\
\mathrm{H} & -1.907427 & 3.205333 \\
\mathrm{H} & -365290 & 4.709773
\end{array}
$$




$\begin{array}{crrc}\mathrm{H} & 1.361696 & 0.384736 & 3.417553 \\ \mathrm{H} & -4.445964 & -3.682656 & -1.807792 \\ \mathrm{H} & -6.458762 & -3.525608 & 0.482125 \\ \mathrm{H} & -6.665789 & -2.789500 & -1.099277 \\ \mathrm{H} & -6.591626 & -4.532106 & -0.945300 \\ \mathrm{H} & -4.133221 & -4.635130 & 1.085255 \\ \mathrm{H} & -4.473270 & -5.674179 & -0.274364 \\ \mathrm{H} & -2.947398 & -4.842545 & -0.200955\end{array}$

\section{$\underline{\text { Iminium ion intermediate }(R, S)-19}$}

Charge $=1$

Multiplicity $=1$

Stoichiometry $=\mathrm{C}_{29} \mathrm{H}_{31} \mathrm{NOF}_{13} \mathrm{Si}$

$E_{\text {elec }}($ gas phase $)=-2841.02861921$ Hartree

$\begin{array}{llll}\text { C } & -1.604951 & 1.134186 & 0.656616\end{array}$

C $\quad-0.486458 \quad 0.887402 \quad-0.124692$

$\begin{array}{llll}\text { C } & 0.017917 & 1.932790 & -0.892732\end{array}$

C $\quad-0.597244 \quad 3.173673 \quad-0.892298$

$\begin{array}{llll}\text { C } & -1.727849 & 3.409799 & -0.126575\end{array}$

$\begin{array}{llll}\text { C } & -2.221054 & 2.376417 & 0.647319\end{array}$

C $\quad 0.117126 \quad-0.524903 \quad-0.168107$

C $\quad-0.463199 \quad-1.333282-1.385445$

N $\quad-1.962661 \quad-1.362245 \quad-1.332533$

C $\quad-2.588364 \quad-0.569423 \quad-2.437346$

C $\quad-1.416482 \quad-0.031300 \quad-3.263377$

C $\quad-0.179211 \quad-0.827277 \quad-2.809153$

C $\quad-2.612380 \quad-2.010265 \quad-0.448821$

C $\quad-4.104996-2.039105-0.312055$

F $\quad-4.302006 \quad-2.318531 \quad 1.064143$

C $\quad-0.066747 \quad 4.228557 \quad-1.805840$

F $\quad-0.519840 \quad 5.466603 \quad-1.489188$

$\begin{array}{llll}\text { C } & -3.465905 & 2.534358 & 1.453040\end{array}$ 


$$
\begin{array}{cccc}
\text { F } & -3.930346 & 3.806503 & 1.463093 \\
\text { C } & 1.656521 & -0.505051 & -0.197273 \\
\text { C } & 2.340582 & 0.423681 & 0.580125 \\
\text { C } & 3.722245 & 0.404943 & 0.666471 \\
\text { C } & 4.464435 & -0.535474 & -0.025787 \\
\text { C } & 3.790658 & -1.461042 & -0.802212 \\
\text { C } & 2.407024 & -1.454853 & -0.880041 \\
\text { C } & 4.536567 & -2.528795 & -1.532542 \\
\text { F } & 4.453312 & -3.730757 & -0.888532 \\
\text { C } & 4.382039 & 1.417245 & 1.544124 \\
\text { F } & 5.734396 & 1.322452 & 1.518576 \\
\text { O } & -0.335166 & -1.261465 & 0.958384 \\
\text { Si } & 0.120602 & -1.721268 & 2.600395 \\
\text { C } & -1.354426 & -2.790845 & 3.127705 \\
\text { C } & 1.719353 & -2.747107 & 2.564779 \\
\text { C } & 0.290626 & -0.170974 & 3.686225 \\
\text { F } & 4.018433 & -2.731072 & -2.780975 \\
\text { F } & 5.853327 & -2.236455 & -1.673222 \\
\text { F } & 3.983838 & 1.272498 & 2.845017 \\
\text { F } & 4.046704 & 2.689288 & 1.184186 \\
\text { C } & -4.803245 & -3.127975 & -1.146175 \\
\text { C } & -6.317589 & -3.057303 & -0.904687 \\
\text { C } & -4.247990 & -4.524928 & -0.833875 \\
\text { F } & -0.443620 & 3.979552 & -3.100564 \\
\text { F } & 1.294394 & 4.263664 & -1.806055 \\
\text { F } & -4.466879 & 1.739528 & 0.946782 \\
\text { F } & -3.291922 & 2.138799 & 2.745594 \\
\text { H } & -2.052621 & -2.557178 & 0.279484 \\
\text { H } & -3.217712 & -1.230006 & -3.012974 \\
\hline & -1.597924 & -0.167110 & -4.319379 \\
\text { H } & -0.718122 & -0.231013 & -2.859671 \\
\hline & -2.351035 & -1.251234 \\
\hline & -1.679480 & -3.458924
\end{array}
$$




$\begin{array}{lrrr}\mathrm{H} & -1.287483 & 1.026016 & -3.097276 \\ \mathrm{H} & -3.194237 & 0.215304 & -2.009080 \\ \mathrm{H} & -1.991151 & 0.358577 & 1.283244 \\ \mathrm{H} & -2.200884 & 4.370172 & -0.129099 \\ \mathrm{H} & 0.898545 & 1.795327 & -1.487712 \\ \mathrm{H} & 1.941313 & -2.201348 & -1.488364 \\ \mathrm{H} & 5.533084 & -0.542434 & 0.033350 \\ \mathrm{H} & 1.800247 & 1.171453 & 1.124438 \\ \mathrm{H} & -1.396670 & -3.716268 & 2.562632 \\ \mathrm{H} & -1.257904 & -3.060123 & 4.174459 \\ \mathrm{H} & -2.300873 & -2.276387 & 3.010358 \\ \mathrm{H} & 1.863870 & -3.207415 & 3.537374 \\ \mathrm{H} & 1.667973 & -3.545481 & 1.833034 \\ \mathrm{H} & 2.597775 & -2.150031 & 2.356787 \\ \mathrm{H} & -0.576704 & 0.475229 & 3.617035 \\ \mathrm{H} & 0.377873 & -0.480298 & 4.723406 \\ \mathrm{H} & 1.174596 & 0.411099 & 3.456480 \\ \mathrm{H} & -4.611986 & -2.900106 & -2.191161 \\ \mathrm{H} & -6.551859 & -3.263954 & 0.130924 \\ \mathrm{H} & -6.719674 & -2.082940 & -1.157456 \\ \mathrm{H} & -6.820933 & -3.792583 & -1.518570 \\ \mathrm{H} & -4.374421 & -4.764525 & 0.213687 \\ \mathrm{H} & -4.781448 & -5.268031 & -1.411235 \\ \mathrm{H} & -3.195957 & -4.620997 & -1.086028 \\ \mathrm{H} & -4.540045 & -1.066777 & -0.487315\end{array}$

\section{References}

(1) Marigo, M.; Wabnitz, T. C.; Fielenbach, D.; Jørgensen, K. A. Angew. Chem. Int. Ed. Engl. 2005, 44, 794.

(2) For 4j see: Luly, J. R.; Dellaria, J. F.; Plattner, J. J.; Soderquist, J. L. J. Org. Chem. 1987, 52, 1487. For 41 see: Phko, P. M.; Aho, J. E. Org. Lett. 2004, 6, 3849.

(3) Juhl, K.; Gathergood, N.; Jørgensen, K. A. Angew. Chem., Int. Ed. 2001, 40, 2995.

(4) Baciocchi, I. J. Am. Chem. Soc. 1967, 89, 4017.

(5) Bøgevig, A.; Juhl, K.; Kumaragurubaran, N.; Zhuang, W.; Jørgensen, K. A. Angew. Chem, Int. Ed. 2002, 41, 1790.

(6) Córdova, A, Watanabe, S.-I.; Tanaka, F.; Notz, W.; Barbas, C. F. III. J. Am. Chem. Soc. 2002, 
124, 1866.

(7) Córdova, A.; Barbas, C. F. III Tetrahedron Lett. 2002, 43, 7749.

(8) Melchiorre, P.; Jørgensen, K. A. J. Org. Chem. 2003, 68, 4151.

(9) Bertelsen, S.; Halland, N. Bachmann, S.; Marigo, M.; Braunton, A.; Jørgensen, K. A. Chem. Commun. 2005, 4821.

(10) Frisch, M. J.; Trucks, G. W.; Schlegel, H. B.; Scuseria, G. E.; Robb, M. A.; Cheeseman, J. R.; Zakrzewski, V. G.; Montgomery, J. A., Jr.; Stratmann, R. E.; Burant, J. C.; Dapprich, S.; Millam, J. M.; Daniels, A. D.; Kudin, K. N.; Strain, M. C.; Farkas, O.; Tomasi, J.; Barone, V.; Cossi, M.; Cammi, R.; Mennucci, B.; Pomelli, C.; Adamo, C.; Clifford, S.; Ochterski, J.; Petersson, G. A.; Ayala, P. Y.; Cui, Q.; Morokuma, K.; Malick, D. K.; Rabuck, A. D.; Raghavachari, K.; Foresman, J. B.; Cioslowski, J.; Ortiz, J. V.; Baboul, A. G.; Stefanov, B. B.; Liu G.; Liashenko, A.; Piskorz, P.; Komaromi, I.; Gomperts, R.; Martin, R. L.; Fox, D. J.; Keith, T.; Al-Laham, M. A.; Peng, C. Y.; Nanayakkara, A.; Gonzalez, C.; Challacombe, M.; Gill, P. M. W.; Johnson, B.; Chen, W.; Wong, M. W.; Andres, J. L.; Gonzalez, C.; Head-Gordon, M.; Replogle, E. S.; Pople, J. A. Gaussian 98, Revision A.7; Gaussian, Inc.: Pittsburgh PA, 1998.

(11) . (b) Frisch, M. J.; et al. Gaussian 03, Revision B.05; Gaussian, Inc.: Pittsburgh PA, 2003. 